\title{
Nonlinear Vibrational Analysis for Integrally Bladed Disk Using Frictional Ring Damper
}

\author{
Yekai Sun, Jie Yuan, Luca Pesaresi and Loïc Salles \\ Department of Mechanical Engineering, Imperial College London \\ London SW7 2AZ, UK \\ E-mail: ys5113@imperial.ac.uk
}

\begin{abstract}
The use of integrally bladed-disk is now very popular in turbomachinery industry since they feature significant aerodynamic and structural improvements along with a significant mass reduction. However, these integrated single structures can arise a major high cycle fatigue issue due to the lack of sufficient damping for dissipating the vibrational energy. This work describes a numerical investigation of the nonlinear dynamic behaviour and nonlinear normal mode for such a bladed-disk with frictional ring damper using the Harmonic Balanced Method (HBM) with alternating Fourier transformation. Jenkins element is used to model the nonlinear contact friction between the disc and ring damper. Using such a modeling strategy, the modal damping and resonance amplitude are directly and efficiently computed through nonlinear normal mode analysis. The initial results show the vibrational level on the blades can be effectively controlled by the parameters of the ring damper model. The effectiveness of ring damper and damping performance is evaluated. This study also indicates the nonlinear normal mode analysis based HBM may be an effective method to analyse the dynamic behaviour of the integrated bladed-disk with frictional ring damper.
\end{abstract}

\section{Introduction}

Blisk is one type of bladed-disk system used in turbomachinery analysis. It is key dynamic components in aircraft engine, which undergoes complicated operational environment with thermal loads, static fluid pressures and rotation-induced centrifugal loads [1]. The aeroelastic excitation on the blisk might cause severe vibration stress leading to high cycle fatigue (HCF). Consequently, $\mathrm{HCF}$ is regarded as a major reason for most of the failures in aero-engine. The flutter effect and mistuned blisk are also potential sources of vibration in the blisk structure [2]. In order to reduce vibration level of blisk, the frictional damper is widely used as main damping source. In a traditional manufacture techniques of blisk, the frictional joints and contact surfaces in assembly point between different components play an important role to dissipate the vibrational energy. Furthermore, friction between contact surfaces is regarded as major damping mechanism, such as friction from under-platform damper and shroud contact surfaces. Frictional joints and contact surfaces have been investigated for past twenty years by many researchers $[3,4,5]$. However, in integrated bladed-disk, the rotor is manufactured in a single piece of component, in stead of an assembled structure with a disk and individual blade. For this reason, the conventional friction joints are no longer favorable due to lack of contact surfaces. The alternative method to introduce contact friction is defined as a ring underneath the blisk wheel. This ring is used as a frictional damper to reduce the vibration of blisk [6]. 
In this paper, the dynamic behaviour of integrally blisk with frictional ring damper is analysed in two aspects, including forced frequency response and nonlinear modal analysis. There are several numerical approaches to directly compute periodic solution for nonlinear dynamic system in time domain or frequency domain. Time integration is traditional time domain method using various schemes, for example, explicit Runge-Kutta scheme, matrix exponential [7] and implicit Newmark scheme [7]. Generally, these methods can provide accurate results but with unaffordable computational costs because small time step is required to capture dynamics behaviour on contact surfaces [8]. Shooting method [9] is another popular method to solve nonlinear problem in time domain by solving boundary values. It provides monodromy matrix as a by-product which can be used for stability analysis. For frequency domain method, Harmonic Balance Method (HBM) is widely used and it discretises the nonlinear property directly with truncated Fourier series [10]. HBM has been extended and improved using various numerical algorithms. HBM with alternating Fourier transformation (AFT) is one of the improved HBM, which is proposed by Cameron in 1989 [11]. In this AFT scheme, nonlinear term is evaluated in time domain and transferred into frequency domain. It provides better approximation than analytical description of nonlinear term in frequency domain. In this work, HBM with AFT is used to solve nonlinear dynamic system.

The nonlinear normal mode (NNM) can be used to describe as an autonomous behaviour of the system. Rosenberg defined the concept of NNM as a vibration unison at the first place in 1960s [12]. For conservative system, the autonomous behaviour of the nonlinear dynamic system is similar to a linear system as a periodic solution. However, in non-conservative nonlinear system, the autonomous response is generally non-periodic and decays with time [1]. In the case of blisk with contact friction, the energy of system is dissipated during the vibration and system is non-conservative due to the friction. Shaw and Pierre proposed a definition of NNM as invariant manifold [13], which provides an accurate solution of NNM even in the case of internal resonance and modal localization. However, modal damping ratio and natural frequency ca not be computed directly. Laxalde et al used a concept of nonlinear complex mode in modal analysis of a non-conservative system [6].This nonlinear complex mode is obtained through solving complex eigen problems. Alternatively, Krack has proposed another NNM definition based on periodic solution by adding a negative artificial damping to compensate the frictional loss [14]. The modal analysis in this work is following Krack's strategy to obtain a periodic solution.

This paper is organised as follows. A detailed description of lumped parameter model and Jenkins element model is presented in Secition 2. Numerical formulations of HBM with AFT, computation of nonlinear normal mode and cyclic symmetric technique are explained in Section 3. Finally, the performance of ring damper is evaluated based on contact condition, damping effectiveness and modal characteristics from numerical results in Section 4.

\section{Model Description}

\subsection{Lumped Parameter Model}

Lumped parameter model has been widely used to investigate the dynamic system at the initial design stage, since it can provide fundamental understanding of the system with low computational costs [15]. In turbomachinery field, several lumped models representing blisk have been designed using a series of mass and spring. The lumped model with ring damper used in this work is referred to existing research by Laxalde et al [16] as shown in Figure 1. There are total four degrees of freedom (DOF) per sector is used to represent the blisk, with two DOFs representing the motion of blade, one DOF for the disk and the other one for the ring damper. There are overall 24 sectors within this cyclic symmetric structure, $N=24$. The model parameters are listed in Table 1. 


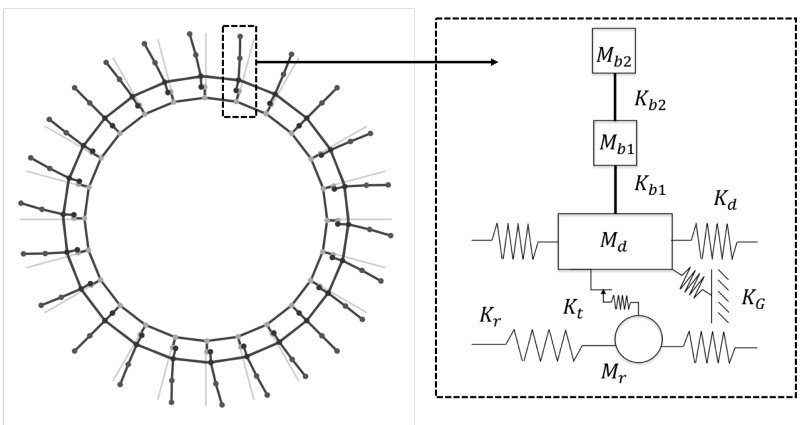

Figure 1. Lumped Parameter Model

Table 1. Lumped Model Parameter

\begin{tabular}{llllllll}
\hline$M_{b 2}$ & $M_{b 1}$ & $M_{d}$ & $K_{b 2}$ & $K_{b 1}$ & $K_{d}$ & $K_{G}$ & $K_{r}$ \\
\hline $0.2 \mathrm{~kg}$ & $0.3 \mathrm{~kg}$ & $1.2 \mathrm{~kg}$ & $2 \cdot 10^{6} \mathrm{~N} / \mathrm{m}$ & $10^{6} \mathrm{~N} / \mathrm{m}$ & $5 \cdot 10^{7} \mathrm{~N} / \mathrm{m}$ & $6 \cdot 10^{5} \mathrm{~N} / \mathrm{m}$ & $2 \cdot 10^{7} \mathrm{~N} / \mathrm{m}$ \\
\hline
\end{tabular}

\subsection{Jenkins Element Model}

Only the friction in tangential direction is considered in this work. The nonlinear force due to friction between the disk and ring damper is computed through a Jenkins element based on Coulomb' law as shown in Figure 2. The relative displacement between disk and ring damper is $x, z$ is internal variable for sliding position. $k_{t}$ is tangential stiffness and $N_{0}$ is normal contact reaction force from rotation of engine. This reaction force is in radial direction about engine rotational axis. Because of normal contact reaction, the ring is assumed to constantly stay in contact with blisk wheel. $N_{0}=m_{r} R \Omega^{2}$, where $m_{r}$ is ring damper mass for each sector, $\Omega$ is engine rotational speed, $R$ is distance between ring damper and rotational axis.

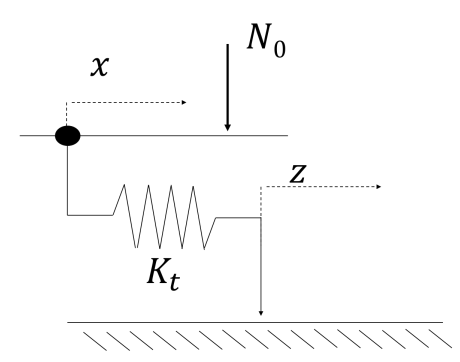

Figure 2. Jenkins Element Model

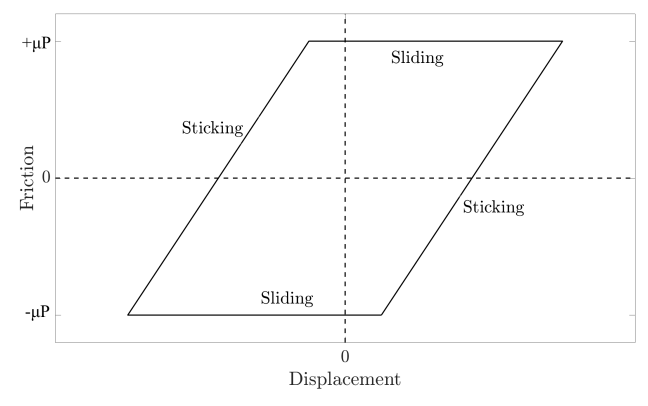

Figure 3. Hysteresis Loop

The Coulomb friction is calculated through Eq.(1), where $\mu$ is static friction coefficient 0.3 and spring stiffness of Jenkins element $K_{t}$ is $5 \cdot 10^{7} \mathrm{~N} / \mathrm{m}$ referred to the parametric study by Schwingshackl et al [17]. There are two contact conditions in Jenkins element model, which are sticking and sliding, as shown in hysteresis loop. In sticking condition, the contact is elastic and no sliding happens. In sliding condition, contact point starts to slide and nonlinear force equals 
to maximum static friction $\mu N_{0}$ against the direction of sliding velocity.

$$
\mathbf{f}_{n l}(t)=\left\{\begin{array}{cl}
K_{t} \times(\mathbf{x}(t)-\mathbf{z}(t)) & \text { if }\left|K_{t}(\mathbf{x}(t)-\mathbf{z}(t))\right| \leq \mu N_{0} \text { (Sticking) } \\
\mu N_{0} \times \operatorname{sign}(\dot{\mathbf{z}}(t)) & \text { if }\left|K_{t}(\mathbf{x}(t)-\mathbf{z}(t))\right|>\mu N_{0} \text { (Sliding) }
\end{array}\right.
$$

\section{Numerical Methods}

The dynamic equation of the system in time domain can be expressed as:

$$
\mathbf{M} \cdot \ddot{\mathbf{x}}(t)+\mathbf{C} \cdot \dot{\mathbf{x}}(t)+\mathbf{K} \cdot \mathbf{x}(t)=\mathbf{f}_{e x}(t)-\mathbf{f}_{n l}(\dot{\mathbf{x}}, \mathbf{x}, t)
$$

Where $\mathbf{M}, \mathbf{K}, \mathbf{C}$ are mass, stiffness and damping matrices respectively. $\mathbf{x}$ is displacement vector including the displacement of four DOFs for all sectors. $\mathbf{f}_{n l}$ is Coulomb friction described in Section 2.2. $\mathbf{f}_{e x}$ is external excitation force applied to blisk in forced response analysis.

To simulate the operating environment of the blisk, the engine order excitation is introduced, which is regarded as an effective traveling wave depending on the number of blades in previous stator. To clarify, a standing wave with constant phase angle on each blisk is used to model this traveling wave, where phase angle depends on the engine order number. The phase angle between the $n^{\text {th }}$ blisk and the first blisk is $\varphi_{n}$ and $E O$ is engine order applied. The excitation force vector is given by Eq.(3). Moreover, this engine order excitation is only applied to two DOFs of blade. The excitation frequency depends on engine order and engine rotational speed, $\omega=E O \times \Omega$.

$$
\begin{gathered}
\varphi_{n}=2 \pi \cdot E O(n-1) / N \\
\left.\mathbf{f}_{e x}^{n}(t)=f_{e x}^{(1)} \times \exp \left[i\left(\omega t-\varphi_{n}\right)\right)\right]
\end{gathered}
$$

\subsection{Harmonic Balance Method with Alternating Fourier Transformation}

Harmonic Balance Method with alternating Fourier transformation is used to solve the nonlinear dynamic equation Eq.(2) in frequency domain. HBM is also known as Fourier-Galerkin method, a continuous property is discretised by Fourier seires. Therefore, $\chi(t)$ representing periodic solution $\mathbf{x}(t)$, nonlinear force $\mathbf{f}_{n l}(t)$ and external excitation $\mathbf{f}_{e x}(t)$ is estimated by Fourier series truncated to $N_{H}$ order of harmonics. $\tilde{\chi}$ is the collection of the all Fourier coefficients for both sine and cosine terms from $0^{t h}$ to $N_{H}^{t h}$ harmonics, where $p$ is the order of harmonics.

$$
\begin{gathered}
\chi(t)=\sum_{p=0}^{N_{H}} \operatorname{real}\left\{\left(\tilde{\chi}^{p, c}+i \cdot \tilde{\chi}^{p, s}\right) \times[\cos (p \omega t)+i \cdot \sin (p \omega t)]\right\} \\
\tilde{\chi}=\left[\tilde{\chi}^{0, c}, \tilde{\chi}^{1, c}+i \tilde{\chi}^{1, s}, \cdots, \tilde{\chi}^{N_{H}, c}+i \tilde{\chi}^{N_{H}, s}\right]^{\mathrm{T}}, \quad \chi \in\left[\mathbf{x}, \mathbf{f}_{e x}, \mathbf{f}_{n l}\right]
\end{gathered}
$$

After implementing Fourier-Galerkin method, the dynamic equation can be expressed as Eq.(5) and Eq.(6), where $\mathbf{A}(\omega)$ is dynamic stiffness matrix in frequency domain shown in Eq.(7). $\mathbf{K}^{p}$, $\mathbf{M}^{p}$ and $\mathbf{C}^{p}$ are cyclic reduced stiffness, mass and damping matrices and will be discussed in details in Section 3.2.

$$
\begin{gathered}
\mathbf{A}(\omega) \times \tilde{\mathbf{x}}=\tilde{\mathbf{f}}_{e x}-\tilde{\mathbf{f}}_{n l}(\tilde{\mathbf{x}}) \\
\mathbf{R}(\tilde{\mathbf{x}}, \omega) \equiv \mathbf{A}(\omega) \times \tilde{\mathbf{x}}-\tilde{\mathbf{f}}_{e x}+\tilde{\mathbf{f}}_{n l}(\tilde{\mathbf{x}})=0 \\
\mathbf{A}(\omega)=\operatorname{diag}\left(\mathbf{A}^{0}, \mathbf{A}^{1}, \mathbf{A}^{2}, \cdots, \mathbf{A}^{N_{H}}\right) \\
\mathbf{A}^{p}=\mathbf{K}^{p}-(p \omega)^{2} \mathbf{M}^{p}+i \times p \omega \mathbf{C}^{p}
\end{gathered}
$$

$\mathbf{R}(\tilde{\mathbf{x}}, \omega)$ is residual of the HBM method expected to be solved iteratively based on NewtonRaphson method. In each iteration, the direct Fourier transformation(DFT) and inverse Fourier transformation(IFT) is alternated to compute $\tilde{\mathbf{f}}_{n l}$. The procedure is demonstrated in Eq.(9).

$$
\tilde{\mathbf{x}} \stackrel{\text { IFT }}{\longrightarrow} \mathbf{x}(t), \dot{\mathbf{x}}(t) \stackrel{\text { Coulomb Friction }}{\longrightarrow} \mathbf{f}_{n l}(\dot{\mathbf{x}}, \mathbf{x}, t) \stackrel{\text { DFT }}{\longrightarrow} \tilde{\mathbf{f}}_{n l}
$$




\subsection{Cyclic Symmetric Techniques}

In order to improve computational efficiency, the cyclic symmetric technique is applied based on the periodicity and cyclic symmetry of the blisk. The detailed description about cyclic symmetry has been demonstrated by Thomas [18]. In his work, he proposed $\mathbf{x}_{R}=\exp (\theta) \mathbf{x}_{L}$, where $\mathbf{x}_{R}$ and $\mathbf{x}_{L}$ are displacement of right and left boundaries. $\theta$ is purely imaginary value measuring the periodicity of mode shape. The periodicity of mode shape depends on harmonic order and engine order in this case. According to Wildheim's theory [19], $\theta$ is product of harmonic order and phase shift of each blisk, $\theta=\varphi_{n} p$. Therefore, the matrix for cyclic symmetric transformation is given in Eq. $(10,11)$.

$$
\begin{gathered}
\Phi_{p}^{E O}=\left[\mathbf{I}, \mathbf{I} \times \exp \left(i p \varphi_{1}\right), \mathbf{I} \times \exp \left(i p \varphi_{2}\right), \cdots, \mathbf{I} \times \exp \left(i p \varphi_{N}\right)\right]^{\mathrm{T}} \\
\Phi^{E O}=\operatorname{diag}\left(\mathbf{I}, \Phi_{1}^{E O}, \Phi_{2}^{E O}, \cdots, \Phi_{N_{H}}^{E O}\right)
\end{gathered}
$$

The $\Phi_{p}^{E O}$ for specific harmonic order $p$ is used to obtained cyclic reduced mass, stiffness and damping matrices in Eq.(7). $\Phi^{E O}$ is globally transformation matrix including harmonic order from 0 to $N_{H}$ and used to computing the cyclic reduced $\tilde{\chi}_{c y c l i c}$ and vice versa. $\mathbf{A}^{+}$is MoorePenrose pseudoinverse matrix of $\mathbf{A}$.

$$
\begin{gathered}
\Lambda_{\text {cyclic }}^{p}=\left(\Phi_{p}^{E O}\right)^{+} \Lambda \Phi_{p}^{E O}, \quad \Lambda \in[\mathbf{M}, \mathbf{K}, \mathbf{C}] \\
\tilde{\chi}_{\text {cyclic }}=\left(\Phi^{E O}\right)^{+} \times \tilde{\chi}
\end{gathered}
$$

\subsection{Nonlinear Normal Mode}

According to Krack' work, a negative artificial damping is introduced into this system [20]. The autonomous system is expressed as Eq.(14), where $\zeta \mathbf{K}$ is an artificial stiffness proportional damping term. $\alpha$ is modal amplitude and $\omega_{0}$ is natural frequency. The same Fourier-Galerkin process in Section 3.1 is applied and residual is given by Eq.(16). Compared to Eq.(6), there are two extra variables $\zeta$ and $\alpha$. Hence, two additional constrians are expected which are mass normalization and phase normalization as shown in Eq.(17, 18). Firstly, mass normalization ensures a positive modal amplitude $\alpha$. Secondly, a phase normalization is required, because absolute phase is arbitrary in autonomous system [14].

$$
\begin{gathered}
\mathbf{M} \cdot \ddot{\mathbf{x}}(t)-(\zeta \times \mathbf{K}) \cdot \dot{\mathbf{x}}(t)+\mathbf{K} \cdot \mathbf{x}(t)+\mathbf{f}_{n l}(\dot{\mathbf{x}}, \mathbf{x}, t)=0 \\
\mathbf{x}(t)=\alpha \sum_{p=0}^{N_{H}} \operatorname{real}\left\{\left(\tilde{\mathbf{x}}^{p, c}+i \cdot \tilde{\mathbf{x}}^{p, s}\right) \times\left[\cos \left(p \omega_{0} t\right)+i \cdot \sin \left(p \omega_{0} t\right)\right]\right\} \\
\mathbf{R}_{1}\left(\tilde{\mathbf{x}}, \omega_{0}, \zeta, \alpha\right)=\mathbf{A}\left(\omega_{0}, \zeta\right) \times \alpha \tilde{\mathbf{x}}-\tilde{\mathbf{f}}_{n l}(\alpha \tilde{\mathbf{x}}) \\
\mathbf{R}_{2}(\tilde{\mathbf{x}})=\tilde{\mathbf{x}}^{\mathrm{T}} \cdot \mathbf{M} \cdot \tilde{\mathbf{x}}-\mathbf{I} \\
\mathbf{R}_{3}(\tilde{\mathbf{x}})=\operatorname{imag}\left(\tilde{\mathbf{x}}_{p=1}^{1}\right)
\end{gathered}
$$

\subsection{Continuation Method}

For forced response analysis, system solution is expected to be computed within a range of excitation frequency $\omega$. In the analysis of NNM, modal characteristics are required within a range of modal amplitude $\alpha$. The continuation method is implemented to track the evolution of system behaviour as varying parameter, $\omega$ or $\alpha$. A proper continuation process requires two steps. At the prediction step, the predicted point is found from previous solution to approximate accurate solution. Then, a new solution is updated from predicted point using Newton's method in correction step. The tangent prediction method and arc-length correction method is selected algorithms for predictor and corrector respectively. The reader can refer to [21] for a detailed description of continuation process. 


\section{Numerical Results}

All natural frequencies (NF) of the lumped parameter model(without the ring damper) are plotted against nodal diameter(ND) in Figure 4. There are three families of mode, where each family of mode is associated with a nodal circle. The modal stiffness of disk increases dramatically with a growing ND [22]. Therefore, disk-dominated modes appear at the mode with relatively larger slop and strong blade-disk coupling, particularly at lower ND. The modes at higher ND tending to a horizontal line are blade-dominated modes with weaker blade-disk coupling. This blade-disk coupling is important because it gives access for communication of vibration energy between blade and disk. There is a veering region between $2^{\text {nd }}$ and $3^{\text {rd }}$ family of mode near ND 3. The modes in veering region tend to show high probability of mode interactions [23]. In order to evaluate the damping performance, the mode at $1^{\text {st }}$ family ND 2 with strong coupling effect is excited. Due to strong bladed-disk coupling, both blade and disk show relatively large displacement when excitation is applied to blade.

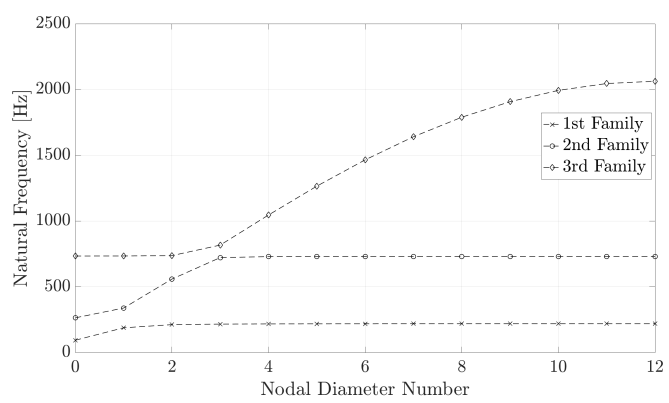

Figure 4. NF / ND Plot

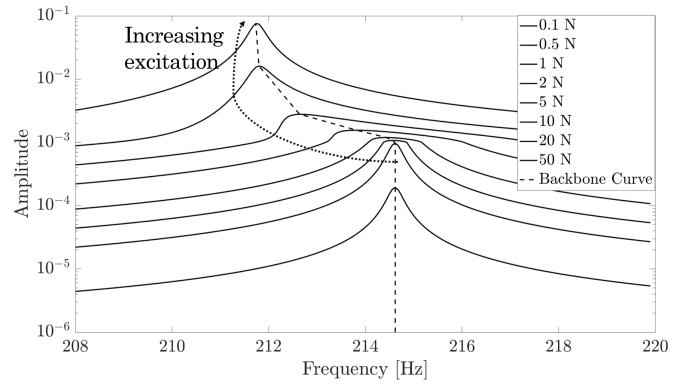

Figure 5. Different Excitation

\subsection{Performance of Ring Damper}

In this section, the damping ability of ring is discussed with the help of frequency response for a forced system. The amplitude of frequency response is recorded from $\mathbf{x}_{b 2}$.

The frequency response with different excitation level is plotted in Figure 5. As can be seen, the magnitude of excitation force affects the contact condition resulting a softening effect. At excitation below $0.5 \mathrm{~N}$, the contact is fully stuck and system shows linear behaviour. With greater excitation level, the displacement of system increases and sliding condition appears. The resonance frequency starts to shift left. When excitation is greater than $50 \mathrm{~N}$, the contact is fully sliding and system becomes linear again with a lower resonance frequency.

The effect of damper mass and engine rotation speed on damping performance is analysed and results are shown in Figure 6 and Figure 7. This suggests that the optimal performance is reached with $30 \mathrm{~g}$ damper mass at rotational speed around $3000 \mathrm{rpm}$. As the increasing damper mass or rotation speed, the resonance peak shift right. The main reason contributing to this phenomenon is nonlinear softening effect as explained above. Normal contact force $N_{0}$ is proportional to the damper mass and squared of rotation speed. For this reason, $N_{0}$ is crucial factor in this case. The contact is fully stuck with larger normal contact force excited by same force level. When the normal contact force decreases, contact starts to slide and the resonance frequency is declining.

\subsection{Modal Characteristics}

During computation of nonlinear mode, the modal characteristics are obtained, including eigenfrequency, mode shape and damping ratio. Eigen-frequency and damping ratio are plotted against system energy shown in Figure 8 and Figure 9. As the system energy increases, natural 


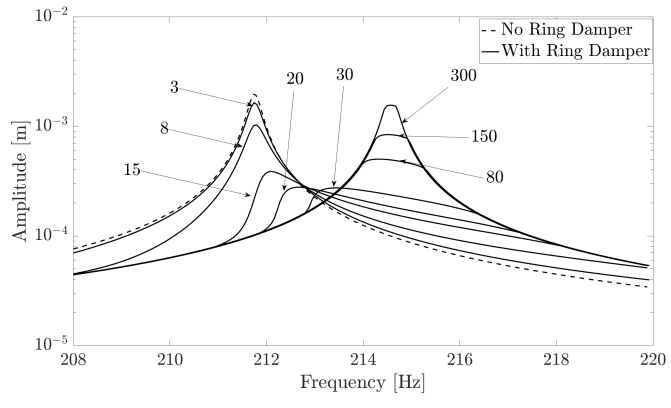

Figure 6. Different Damper Mass [g]

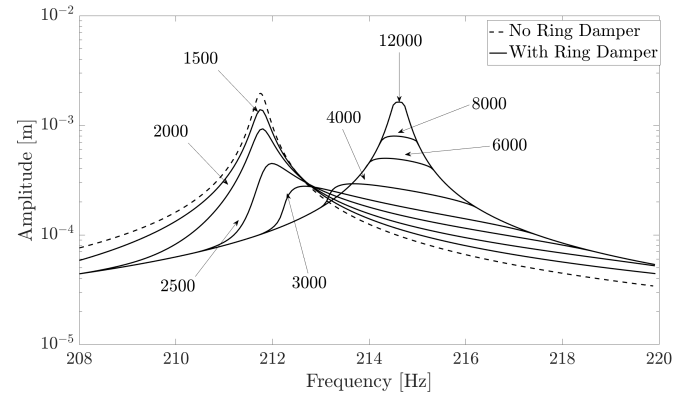

Figure 7. Different Rotation Speed [rpm]

frequency is decreasing due to softening effect caused by friction, which corresponds to the backbone curve in Figure 5. In terms of damping ratio, it stays at zero with lower system energy. At this point, the contact is fully stuck. It follows that there is no energy dissipation due to friction. At the transition from sticking to sliding, damping ratio reaches its maximum point and ring damper provides the optimal damping performance. When system energy is larger enough, damping ratio begins to drop because of fully sliding contact. The damping ratio will return to zero asymptotically with infinity system energy.

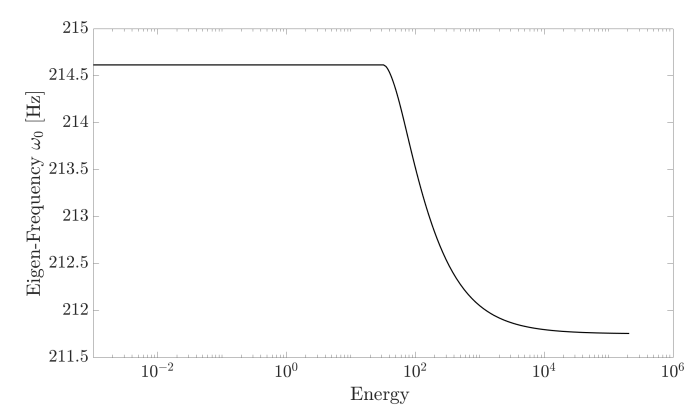

Figure 8. Frequency Energy Plot

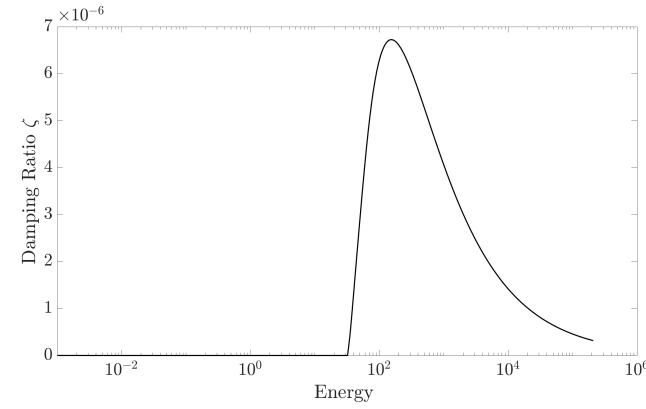

Figure 9. Damping Ratio / Energy

\section{Conclusion}

In summary, a lumped parameter model with Jenkins element model is used to simulate the nonlinear dynamic behavior of blisk with a frictional ring damper. This nonlinear dynamic system is analysed through forced frequency response and nonlinear modal analysis. The numerical results provided a qualitative analysis for nonlinear dynamic behaviour of the blisk with frictional ring damper.

Generally, both effectiveness and limitations of this ring damper are clearly pointed out from the analysis. The performance of the damper is highly depending on the relative motion between disk and ring. Ring damper is able to provide considerable damping when relative displacement is large. In other words, if a mode with weak bladed-disk coupling effect is excited, the motion of disk is relatively small and ring damper will show less effectiveness. In addition, the normal contact reaction force is also crucial for damping effect. In a sense, if the ring is well designed for blisk, this damping technique can be regarded as an alternative to classical friction joints.

In terms of numerical methodology, modal damping ratio and natural frequency are directly and efficiently computed within modal analysis. Forced response analysis provides corresponding 
results to the modal characteristics. Finally, this paper indicates that nonlinear normal mode is an efficient way to study dynamic behaviour of blisk with frictional ring damper.

\section{Acknowledgement}

The first author is grateful to China Scholarship Council (File NO. 201708060239) for providing the financial support for this project.

\section{References}

[1] Krack M, Salles L and Thouverez F, 2016, Vibration Prediction of Bladed Disks Coupled by Friction Joints, Archives of Computational Methods in Engineering, 24, pp. 589-636

[2] Griffin J H and Sinha A, 1985, The Interaction between Mistuning and Friction in the Forced Response of Bladed Disk Assemblies, Journal of Engineering for Gas Turbines and Power, 107, pp. 205-211.

[3] Griffin J H, 1990, A Review of Friction Damping of Turbine Blade Vibration, International Journal of Turbo and Jet Engines, 7, pp. 297-307.

[4] Pesaresi L, Salles L, Jones A, Green J S and Schwingshackl C W, 2017, Modelling the Nonlinear Behaviour of an Underplatform Damper Test Rig for Turbine Applications, Mechanical Systems and Signal Processing, 85, pp. 662-679.

[5] Armand J, Pesaresi L, Salles L and Schwingshackl C W, 2016, A Multiscale Approach for Nonlinear Dynamic Response Predictions With Fretting Wear, Journal of Engineering for Gas Turbines and Power, 139(2), pp. 022505-022505-7

[6] Laxalde D, Salles L, Blanc L and Thouverez F, and Lombard J P, 2008, Non-Linear Modal Analysis for Bladed Disks With Friction Contact Interfaces, Proceedings of ASME Turbo Expo 2008, 5 (4), pp. 456-467.

[7] Cardona A, Lerusse A and Géradin M, 1998, Fast Fourier Nonlinear Vibration Analysis, Computational Mechanics, 22, pp. 128-142

[8] Yuan J, El-Haddad F, Salles L and Wong C, 2018, Numerical Assessment of Reduced Order Modeling Techniques for Dynamic Analysis of Jointed Structures with Contact Nonlinearities, Proceedings of ASME Turbo Expo 2018, Accepted.

[9] Kerschen G, 2014, Modal Analysis of Nonlinear Mechanical Systems, (Vienna: Springer), pp. 215-250.

[10] Detroux T, Renson L, Massetand L and Kerschen G, 2015, The Harmonic Balance Method for Bifurcation Analysis of Large-scale Nonlinear Mechanical Systems, Computer Methods in Applied Mechanics and Engineering, 296, pp. 18-38.

[11] Cameron T M and Griffin J H, 1989, An Alternating Frequency/Time Domain Method for Calculating the Steady-state Response of Nonlinear Dynamic Systems, Journal of Applied Mechanics.

[12] Rosenberg R M, 1960, Normal Modes of Nonlinear Dual-mode Systems, Journal of Applied Mechanics, 27, pp. $263-268$

[13] Shaw S W and Pierre C, 1991, Non-linear Normal Modes and Invariant Manifolds, Journal of Sound and Vibration, 150(1), pp. 170-173

[14] Krack M, 2014, The Concept of Nonlinear Modes Applied to Friction-Damped Systems (Doctoral Thesis), Leibniz Universität Hannover

[15] Yuan J, Fabrizio S, Branislav T, Giuliano A, Sophoclis P and Ramesh R, 2017, Novel Frame Model for Mistuning Analysis of Bladed Disk Systems, Journal of Vibration and Acoustics, 139, pp. 031016-031016-13

[16] Laxalde D, Sinou J J, Thouverez F, and Lombard J P, 2007, Qualitative Analysis of Forced Response of Blisks with Friction Ring Dampers, European Journal of Mechanics A/Solids, 26 (4), pp. 676-687.

[17] Schwingshackl C W, Petrov E P and Ewins D J, 2012, Effects of Contact Interface Parameters on Vibration of Turbine Bladed Disks With Underplatform Dampers, Journal of Engineering for Gas Turbines and Power, 134(3), pp. 032507-032507-8

[18] Thomas D L, 1974, Standing Waves in Rotationally Periodic Structures, Journal of Sound and Vibration, 37, pp. $288-290$

[19] Wildheim S, 1979, Excitation of Rotationally Periodic Structures, Transaction of ASME, 46, pp. 878-883

[20] Krack M, 2015, Nonlinear Modal Analysis of Nonconservative Systems: Extension of the Periodic Motion Concept, Computers and Structures, 154, pp. 59-71

[21] Sarrouy E and Sinou J J, 2011, Advances in Vibration Analysis Research (Rijeka: nTech), pp. 419-434

[22] Castanier M P and Pierre C, 2006, Modeling and Analysis of Mistuned Bladed Disk Vibration: Current Status and Emerging Directions, Journal of Propulsion and Power, 22(2), pp. 384-396

[23] Kenyon J, Griffin J and Kim N, 2004, Frequency Veering Effects on Mistuned Bladed Disk Forced Response, Journal of Propulsion and Power, 20(5), pp. 863-870 